\title{
Automated MRI-based volumetry of basal ganglia and thalamus at the chronic phase of cortical stroke
}

\author{
Cindy Baudat ${ }^{1}$ (D) Bénédicte Maréchal ${ }^{1,2}$ (D) Ricardo Corredor-Jerez ${ }^{1,2}$ (D) \\ Tobias Kober $^{1,2}$ (D) - Reto Meuli ${ }^{1}$ (D) Patric Hagmann ${ }^{1}$ (D) Patrik Michel $^{3}$ (D) \\ Philippe Maeder ${ }^{1}$ (D) V Vincent Dunet ${ }^{1}$ (D)
}

Received: 21 April 2020 / Accepted: 10 June 2020 / Published online: 17 June 2020

(C) The Author(s) 2020

\begin{abstract}
Purpose We aimed at assessing the potential of automated MR morphometry to assess individual basal ganglia and thalamus volumetric changes at the chronic phase after cortical stroke.

Methods Ninety-six patients (mean age: $65 \pm 18$ years, male 55) with cortical stroke at the chronic phase were retrospectively included. Patients were scanned at $1.5 \mathrm{~T}$ or $3 \mathrm{~T}$ using a T1-MPRAGE sequence. Resulting 3D images were processed with the MorphoBox prototype software to automatically segment basal ganglia and thalamus structures, and to obtain $Z$ scores considering the confounding effects of age and sex. Stroke volume was estimated by manual delineation on T2-SE imaging. $Z$ scores were compared between ipsi- and contralateral stroke side and according to the vascular territory. Potential relationship between $Z$ scores and stroke volume was assessed using the Spearman correlation coefficient.

Results Basal ganglia and thalamus volume $Z$ scores were lower ipsilaterally to MCA territory stroke $(p$ values $<0.034)$ while they were not different between ipsi- and contralateral stroke sides in non-MCA territory stroke ( $p$ values $>0.37)$. In MCA territory stroke, ipsilateral caudate nucleus (rho $=-0.34, p=0.007$ ), putamen (rho $=-0.50, p<0.001$ ), pallidum (rho $=-0.44$, $p<0.001$ ), and thalamus (rho $=-0.48, p<0.001)$ volume $Z$ scores negatively correlated with the cortical stroke volume. This relation was not influenced by cardiovascular risk factors or time since stroke.

Conclusion Automated MR morphometry demonstrated atrophy of ipsilateral basal ganglia and thalamus at the chronic phase after cortical stroke in the MCA territory. The atrophy was related to stroke volume. These results confirm the potential role for automated MRI morphometry to assess remote changes after stroke.
\end{abstract}

Keywords Basal ganglia $\cdot$ Brain morphometry $\cdot$ Magnetic resonance imaging $\cdot$ Stroke $\cdot$ Thalamus

\section{Abbreviations}

ACA Anterior cerebral artery

ADNI Alzheimer's disease neuroimaging initiative

CNR Contrast-to-noise ratio

MCA Middle cerebral artery

Vincent Dunet

Vincent.Dunet@chuv.ch

1 Department of Diagnostic and Interventional Radiology, Lausanne University Hospital and University of Lausanne, Rue du Bugnon 46, CH-1011 Lausanne, Switzerland

2 Advanced Clinical Imaging Technology, Siemens Healthcare AG, Lausanne, Switzerland

3 Stroke Center, Neurology Service, Department of Clinical Neurosciences, Lausanne University Hospital and University of Lausanne, Lausanne, Switzerland
PCA Posterior cerebral artery

\section{Introduction}

Stroke is the second largest cause of mortality and the second most common cause of disability-adjusted life-years worldwide, after ischemic heart disease $[1,2]$. The World Health Organization estimated that 13.7 million people suffered from a stroke in 2016, of which 5.5 million died. After a stroke, brain plasticity manifests itself as remote changes of the brain, of which some reflect interconnection degeneration and others could counterbalance stroke-induced deficits [3-5]. Identification and quantification of remote changes of the brain are thus of interest in order to find imaging markers for therapy efficacy assessment at the patient level. 
In the past decades, automated morphometry of brain magnetic resonance imaging (MRI) has emerged and currently represents a promising tool in clinical practice. Some studies based on morphometric techniques using MRI images led to the better understanding of the mechanisms underlying brain plasticity and functional recovery [6-9]. However, the techniques used for morphometry remain mainly based on group comparisons and are complex to implement in daily practice. These imaging techniques are likely to mask some of the intersubject variabilities essential to the evaluation of individual brain plasticity. The automated brain MRI segmentation prototype software called MorphoBox has recently been developed to provide volumetric data at the patient level [10]. The measurements obtained with this fully automated algorithm have demonstrated excellent correlation with those obtained with other validated algorithms used for brain morphometry, including FreeSurfer and Statistical Parametric Mapping [10]. It has also shown similar or better performance for the individual classification of patients with cognitive impairment [10] as well as to differentiate patients with Parkinson's disease from patients with vascular parkinsonism based on their individual brain morphometry [11]. Basal ganglia and thalamus are key anatomical and functional nodes for tracts coming from or going to the cortex, and are thus particularly exposed to remote changes after a cortical injury, such as stroke [4]. Nevertheless, the feasibility and utility of assessing remote changes in basal ganglia and thalamus after cortical stroke with MorphoBox remain to be demonstrated.

The main goal of this work was therefore to evaluate the potential of automated brain MRI morphometry for the detection of volume changes in basal ganglia and thalamus, in patients with chronic stroke. Secondary aims were to evaluate the impact of stroke side, vascular territory, stroke volume, cardiovascular risk factors, and time since stroke on these changes.

\section{Methods}

\section{Study design}

In this single-center retrospective study, patients referred to the Department of Diagnostic and Interventional Radiology of our institution for a brain MRI between January 2010 and August 2018 with a diagnosis of cortical stroke at the chronic phase were searched on our institutional Radiology Information System and consecutively enrolled. Chronic stroke was defined according to current recommendations as stroke more than 3 weeks old [12]. Inclusion criteria were as follows: age $>18$ years, single vascular territory stroke involving cortex, stroke size $>2 \mathrm{~cm}$. Exclusion criteria were as follows: associated acute stroke, posterior fossa stroke, lacunar or basal ganglia-thalamus stroke, history of chronic neurodegenerative brain disease (e.g., Alzheimer's disease, multiple sclerosis), severe traumatic brain injury, or brain tumor.

For each subject included in the study, the side (right or left) and the vascular territory (anterior cerebral artery [ACA], middle cerebral artery $[\mathrm{MCA}]$, or posterior cerebral artery [PCA]) affected by the stroke were collected as well as stroke etiology and cardiovascular risk factors (hypertension, diabetes, smoking, dyslipidemia). All collected data were anonymized to comply with national ethical guidelines. Therefore, patients' consent was waived, and the study was conducted in accordance with the World Medical Association Declaration of Helsinki.

\section{MR imaging acquisition}

All patients selected according to the study criteria underwent brain MRI (MAGNETOM Aera, Symphony, Skyra, Skyra fit, Prisma fit, Vida or Verio, all Siemens Healthcare, Erlangen, Germany) at 1.5 -Tesla (14\%) or 3-Tesla (86\%) in the Department of Diagnostic and Interventional Radiology of our institution. The MRI protocol included a 3D sagittal T1 magnetization prepared rapid gradient echo (T1-MPRAGE) sequence using the $1.5 \mathrm{~T}$ and $3 \mathrm{~T}$ Alzheimer's disease neuroimaging initiative (ADNI) protocols [13-15] and a 2D axial T2 spin echo (T2-SE) sequence (TR $5000 \mathrm{~ms}$, TE $77 \mathrm{~ms}$, flip angle $150^{\circ}$, matrix $256 \times 240$, slice thickness $3 \mathrm{~mm}$, slice number 43 , voxel size $0.5 \times 0.5 \times 3 \mathrm{~mm}^{3}$ ).

\section{MR imaging analysis}

MRI analysis was independently performed for T1-MPRAGE and T2-SE sequences. The MPRAGE sequence of each participant was segmented using the automated MorphoBox prototype software [10] to obtain absolute volumes in milliliters $(\mathrm{mL})$ of the following structures for each individual: total intracranial volume (TIV); right and left basal ganglia and thalamus (Fig. 1). These volumes were also expressed as percentage (\%) of the TIV and compared to volumes obtained in a population of healthy control subjects included in the MorphoBox tool. This control population corresponds to a subset of healthy subjects included in the ADNI study [15, 16] with additional younger healthy subjects added from inhouse acquisitions following ADNI guidelines. In total, it consists of 303 healthy controls (mean age $66 \pm 19$ years, age range 19-90 years, $51 \%$ of healthy male) who underwent 3 Tesla brain MRI using the standardized ADNI protocol. Normative ranges were calibrated on this population using a log-linear regression model, taking into account the confounding effects of age and sex as covariates. Deviations from normative ranges of individual volume estimates were assessed by $Z$ scores. For every patients, voxel size in cubic millimeters was recorded, as well as two global contrast-to-noise 
measurements computed by MorphoBox as image segmentation by-products, respectively between gray matter and white matter (CNRgw) and between gray matter and cerebrospinal fluid (CNRgc).

Axial T2-SE images were analyzed using Carestream software (Version 12.1.6.0117, Carestream Health, Toronto, Canada). The volume of cerebral infarction was manually delineated by a single neuroradiologist with 10 years of experience in brain imaging. A region of interest (ROI) was drawn on each axial section on which the cerebral infarction was visible. The sum of the ROI areas in square millimeters was then multiplied by the nominal slice thickness in millimeters to obtain an estimate of the volume of cerebral infarction in cubic millimeters and in milliliters (Fig. 2). This measure was performed twice for 20 patients randomly chosen to assess estimation reliability. Fazekas score was also recorded to take into account white matter hyperintense lesions, which are a marker of small vessel disease [17].

\section{Statistics}

Statistical analyses were conducted with STATA software (Version 16.0, StataCorp, College Station, Texas, USA). In order to assess the potential correlation between stroke volume and basal ganglia $Z$ scores, we performed a sample size calculation. Considering that we aimed to detect a negative correlation of at least -0.40 with $80 \%$ power using a onesided $5 \%$ level test, we estimated a minimum sample size of 47 patients. Continuous variables (age, structure volumes, and $Z$ scores, stroke volumes) were expressed as mean \pm standard deviations (SD). Categorical variables (sex, side, and vascular territory affected by the stroke, cardiovascular risk factors) were expressed as numbers or percentages. Two groups were defined according to the vascular territory affected by the stroke: strokes affecting the territory of the MCA versus those affecting other vascular territories (i.e., ACA or PCA), referred as "non-MCA" in the rest of the manuscript. This segregation was chosen because the thalamus, putamen, and pallidum are closely connected to the vascularized regions by the MCA. Stroke location distribution between right and left hemispheres and between vascular territories was compared using Fisher's exact test. Reliability of stroke volume measurement was assessed using Lin's test. Absolute volumes of strokes, as well as absolute volumes, relative volumes, and $Z$ scores of basal ganglia and thalamus were compared between the ipsilateral and contralateral stroke sides and according to the vascular territory affected by the stroke using the Wilcoxon rank-sum test. Potential relation between basal
Fig. 1 Exemplary segmentation of a MPRAGE sequence with the MorphoBox prototype. From the T1 image (a), the algorithm extracts the total brain volume and then segments the different structures (b) to obtain a map of the Z-scores (c) in comparison with a population of healthy subjects considering age and sex. The final morphometric ratio normalized by the total intracranial volume appears in the form of a Table (d) showing atrophy of the left basal ganglia and thalamus in this case of a left MCA stroke (frontal atrophy of the gray and white matter is also seen in orange on image c)






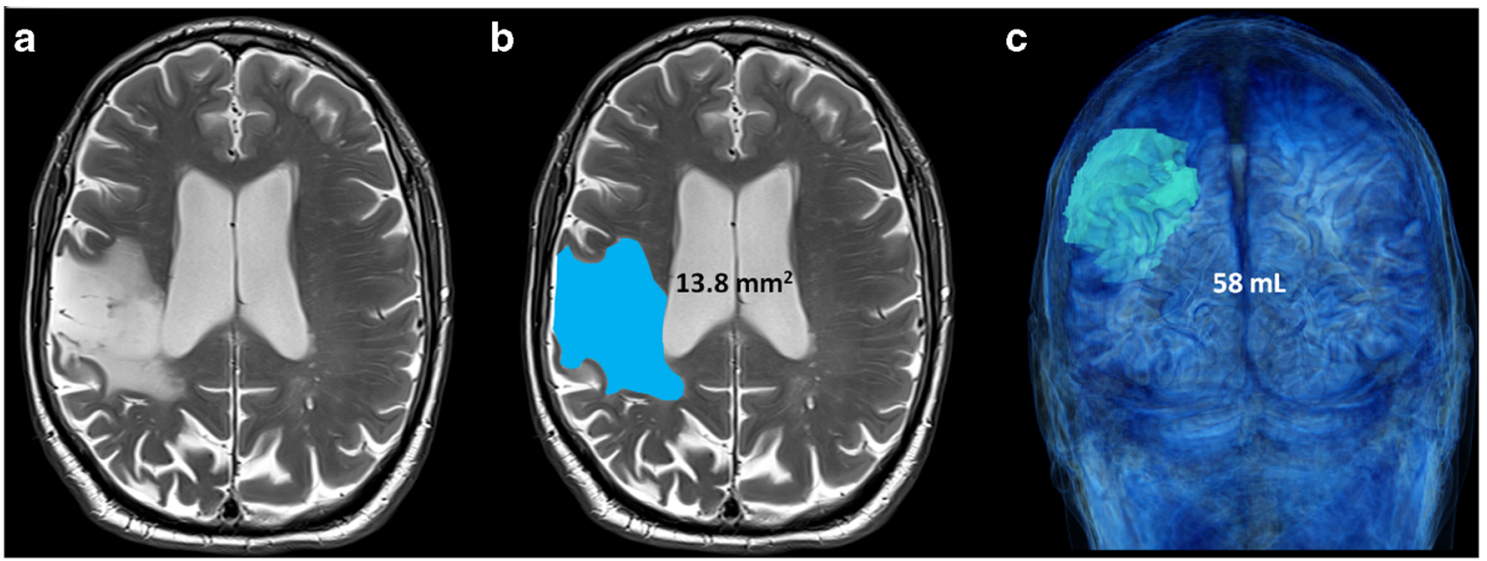

Fig. 2 Illustration of the manual segmentation of a right sylvian cortical stroke. On the T2-SE sequence in axial section (a), the surface of the cortical stroke was delimited section by section (b) and then multiplied

ganglia and thalamus $Z$ scores and stroke volumes was assessed using the non-parametric Spearman's correlation coefficient (rho) and linear regression analysis. Voxel size,

Table 1 Patients' characteristics

\begin{tabular}{ll}
\hline Variables & Median [IQR] or number \\
\hline Sex (male/female) & $56 / 42$ \\
Age (years) & $69[57-78]$ \\
Weight $(\mathrm{kg})$ & $73.2[64.2-83.0]$ \\
BMI $\left(\mathrm{kg} / \mathrm{m}^{2}\right.$ ) & $26.0[22.9-28.4]$ \\
Cardiovascular risk factors & \\
Smoking (past/present/no) & $16 / 26 / 56$ \\
Hypertension (yes/no) & $67 / 31$ \\
Dyslipidemia (yes/no) & $63 / 35$ \\
Diabetes (yes/no) & $21 / 77$ \\
Fibrillation (yes/no) & $14 / 84$ \\
Myocardial infarct (yes/no) & $15 / 83$ \\
Peripheral artery disease (yes/no) & $8 / 90$ \\
Stroke etiologies & \\
Atherosclerosis & 41 \\
bEmbolic & 10 \\
Dissection & 5 \\
Vasculitis & 6 \\
Unkown & 36 \\
Stroke location & 14 \\
Anterior cerebral artery & 62 \\
Middle cerebral artery & 22 \\
Posterior cerebral artery & \\
Vascular burden & \\
Fazekas score (0/1/2/3) & \\
Carotid artery stenosis $\geq 50 \%$ (yes/no) & $15 * / 83$ \\
\hline
\end{tabular}

*Ten patients with a MCA stroke and 5 patients with a non-MCA stroke had a carotid artery stenosis $\geq 50 \%$. None had an occlusion by the nominal section thickness to obtain the volume of the stroke, visible on the volume rendering image (c)

CNRgw, and CNRgc were used as covariates to take into account potential bias due to inter-scan variability. Age and delay from stroke onset to MRI were also used as covariates to take into account inter-subject variability. The value of the rho coefficient was interpreted using the Landis and Koch scale, according to the following categorization: very low correlation if rho $<|0.20|$; low if rho: $|0.21-0.40|$; moderate if rho: $|0.41-0.60|$; strong if rho: $|0.61-0.80|$, and excellent if rho $>|0.80|$. Finally, multivariate linear regression analysis was performed to assess the impact of cardiovascular risk factors (age, hypertension, smoking, diabetes, dyslipidemia), Fazekas score, carotid artery stenosis $\geq 50 \%$, and delay from stroke onset on this interrelation. For all statistics, the determined $p$ value was considered statistically significant for values $<0.05$.

\section{Results}

\section{Study population}

Overall, 98 patients (mean age: $65 \pm 18$ years, female 42 , male 56) met the inclusion and exclusion criteria between January 2010 and August 2018. Patients' characteristics are summarized in Table 1. Two patients were subsequently excluded from volumetric analysis due to poor image quality impeding the manual delineation of the stroke volume on T2-SE images or automated segmentation of MPRAGE. Basal ganglia, thalamus, and stroke volumes were thus obtained for 96 patients (mean age $65 \pm 18$ years, female 41 , male 55 ). The mean delay between stroke onset and MRI evaluation was $73.1 \pm$ 93.7 months (range 1.3-427 months).

Of the 96 patients retained in the volumetric study, 52 had a right-sided stroke and 44 had a left-sided stroke. Regarding the vascular territory, 13 had a stroke in the territory of the ACA, 61 patients had a stroke affecting the territory of the MCA, and 22 had a stroke in the territory of the PCA. There 




Fig. 3 Exemplary segmentation of the MPRAGE sequence in patients with small and large cortical strokes. Small (top row) and large (bottom row) cortical strokes are displayed on axial T2-SE images (left column) along with axial T1 MPRAGE view, segmentation and $Z$ score maps. The small cortical stroke measured $8 \mathrm{~mL}$ and the large measured $47 \mathrm{~mL}$. Resulting ipsilateral caudate nucleus, putamen, pallidum, and thalamus $Z$ scores were $2.10,-0.07,1.24,2.01$ and $-1.37,-5.09,-3.73$, and 3.34 , respectively was no significant difference in stroke distribution regarding the side and vascular territory $(p=0.69)$. The mean stroke volume was $29.7 \pm 42.3 \mathrm{~mL}$, with no significant difference according to stroke side or vascular territory $(p>0.46)$. The stroke volume in MCA territory was not significantly different from the stroke volume in non-MCA territory $(34.5 \pm 51.3 \mathrm{~mL}$ versus $21.4 \pm 15.4 \mathrm{~mL}, p=0.15)$. Lin's test demonstrated excellent reliability of the stroke volume estimation (Pearson's rho $=0.999$, rho $\_c=0.996, \mathrm{Cb}=0.997$, mean difference -1 $\pm 3.5 \mathrm{~mL}, 95 \% \mathrm{CI}-7.9-5.9 \mathrm{~mL})$. Time since stroke was similar between patients with MCA and non-MCA stroke $(p=$ $0.63)$.

\section{Volume of basal ganglia and thalamus}

MPRAGE scans of all 96 included patients were successfully segmented by the morphometry software. This corresponds to a feasibility of $98 \%$ (i.e., 96 of 98 initially enrolled patients). The individual duration of post-processing of the T1MPRAGE sequences was 2-3 min for each participant (i.e., approximately $5 \mathrm{~h}$ in total for all included subjects). Figure 3 displays examples of segmentation in two patients with a small and large cortical stroke, respectively.

Absolute volumes, relative volumes, and $Z$ scores of the basal ganglia and thalamus are summarized in Table 2 according to the ipsilateral or contralateral side of the stroke regardless of the affected vascular territory. Overall, the putamen had a significantly lower absolute volume, relative volume, and $Z$ score on the side affected by the stroke. The thalamus had also lower relative volume and $Z$ score ipsilaterally to the stroke while the pallidum had a lower $Z$ score ipsilaterally to the stroke. The caudate nucleus was not significantly different between the two sides.

$Z$ scores are presented in Table 3 for the MCA and nonMCA territories. For patients with stroke in the MCA territory, the $Z$ scores of ipsilateral basal ganglia and thalamus were lower compared to the contralateral side (all $p<0.034$ ). In stroke affecting non-MCA territories, the $Z$ scores of basal ganglia and thalamus were not significantly different between the ipsilateral and contralateral side (all $p>0.37$ ).

\section{Correlation between stroke volume and $Z$ scores of the basal ganglia and thalamus}

Overall, there was a negative correlation between stroke volume and ipsilateral putamen $(\mathrm{rho}=-0.39, p<0.001)$, pallidum (rho $=-0.27, p=0.007$ ), and thalamus (rho $=-$ $0.29, p=0.003$ ) volume $Z$ scores but not with contralateral basal ganglia and thalamus volume $Z$ scores (all $p>0.098$ ). Considering the vascular territory affected by the stroke, there 
Table 2 Basal ganglia and thalamus volumes comparison. Absolute volumes, relative volumes, and $Z$ scores were compared between ipsi and contralateral sides of the stroke, regardless of the vascular territory, using the Wilcoxon rank sum test. TIV total intracranial volume

\begin{tabular}{llll}
\hline Volume in $\mathrm{mL}$ & \multicolumn{2}{l}{$\begin{array}{l}\text { Ipsilateral to stroke } \\
n=96\end{array}$} & $\begin{array}{l}\text { Contralateral to stroke } \\
n=96\end{array}$ \\
\hline Caudate nucleus & $4.46 \pm 0.87$ & $4.59 \pm 0.84$ & 0.14 \\
Putamen & $6.08 \pm 1.21$ & $6.50 \pm 0.90$ & 0.027 \\
Pallidum & $1.71 \pm 0.34$ & $1.79 \pm 0.30$ & 0.15 \\
Thalamus & $6.36 \pm 1.06$ & $6.67 \pm 0.97$ & 0.058 \\
\% of the TIV & & & \\
Caudate nucleus & $0.32 \pm 0.05$ & $0.33 \pm 0.05$ & 0.13 \\
Putamen & $0.44 \pm 0.08$ & $0.47 \pm 0.06$ & 0.018 \\
Pallidum & $0.12 \pm 0.02$ & $0.13 \pm 0.02$ & 0.077 \\
$\quad$ Thalamus & $0.46 \pm 0.06$ & $0.48 \pm 0.05$ & 0.016 \\
$Z$ score & & & \\
Caudate nucleus & $0.16 \pm 1.34$ & $0.48 \pm 1.26$ & 0.085 \\
Putamen & $-0.90 \pm 1.84$ & $-0.22 \pm 1.29$ & 0.013 \\
Pallidum & $-0.53 \pm 1.53$ & $-0.03 \pm 1.17$ & 0.025 \\
Thalamus & $-0.61 \pm 1.52$ & $0.09 \pm 1.32$ & 0.001 \\
\hline
\end{tabular}

was no significant relation between the stroke volume and $Z$ scores of ipsilateral or contralateral basal ganglia and thalamus for non-MCA strokes (all $p>0.09$ ). In patients with MCA stroke, the stroke volume was significantly correlated with the $Z$ scores of ipsilateral caudate nucleus (rho $=-0.34, p=$ 0.007 ), putamen (rho $=-0.50, p<0.001$ ), pallidum (rho $=-$ $0.44, p<0.001$ ), and thalamus (rho $=-0.48, p<0.001$ ) (Fig. 4), but not with contralateral $Z$ scores (all $p>0.28$ ). On multivariate analysis, the putamen (beta $=-0.61, p<0.001$ ), pallidum (beta $=-0.38, p=0.004$ ), and thalamus (beta $=-$ $0.38, p=0.003) Z$ scores were independently correlated with

Table $3 Z$ scores of basal ganglia and thalamus volumes according to vascular territories. $Z$ scores were compared between ipsi and contralateral sides of the stroke, according to the vascular territory affected by the stroke, using the Wilcoxon rank sum test. MCA middle cerebral artery

\begin{tabular}{llll}
\hline Z scores & Ipsilateral to stroke & Contralateral to stroke & $p$ value \\
\hline MCA stroke & $n=61$ & $n=61$ & \\
Caudate nucleus & $-0.03 \pm 1.29$ & $0.50 \pm 1.20$ & 0.034 \\
Putamen & $-1.21 \pm 1.92$ & $-0.17 \pm 1.26$ & 0.002 \\
Pallidum & $-0.67 \pm 1.66$ & $0.10 \pm 1.17$ & 0.012 \\
Thalamus & $-0.72 \pm 1.59$ & $0.22 \pm 1.35$ & 0.001 \\
Non-MCA stroke & $n=35$ & $n=35$ & \\
Caudate nucleus & $0.48 \pm 1.40$ & $0.42 \pm 1.37$ & 0.90 \\
Putamen & $-0.35 \pm 1.57$ & $-0.31 \pm 1.35$ & 0.96 \\
Pallidum & $-0.30 \pm 1.27$ & $-0.25 \pm 1.18$ & 0.76 \\
Thalamus & $-0.40 \pm 1.37$ & $-0.13 \pm 1.28$ & 0.37 \\
\hline
\end{tabular}

the stroke volume but not with cardiovascular risk factors, Fazekas score, presence of carotid artery stenosis $\geq 50 \%$, or delay from stroke onset (all $p>0.24$ ).

\section{Discussion}

The main results of the study can be summarized as follows: (1) Individual automated brain morphometry of patients with cortical stroke was feasible in $98 \%$ of our cases; (2) Ipsilateral caudate nucleus, putamen, pallidum, and thalamus were atrophic in patients affected by MCA stroke only; (3) Atrophy of ipsilateral caudate nucleus, putamen, pallidum, and thalamus were negatively correlated with stroke volume. These results indicate that automated brain morphometry in MRI is feasible and can detect remote changes induced by cortical stroke at the chronic phase at the patient level. These changes are influenced by cortical stroke location and extent, but not by cardiovascular risk factors and time since stroke.

\section{Feasibility of individual morphometry in patients with cortical stroke}

Cerebral MR morphometric assessment has undergone significant development over the last 20 years [18]. It allows detecting morphological changes related to congenital or acquired diseases, psychiatric, neurodegenerative, or related to other etiologies. Several morphometric methods are available such as voxel-based analyses, which allow the detection of subtle changes in trophicity, cortical thickness, or the gyri. Nevertheless, these methods are based on a long postprocessing of the acquired images and mostly on the comparison between a group of interest and a control group, not giving access in real time to the quantitative analysis in single subjects. The study proceeded to a volume-based morphometry analysis at the patient level rapidly generating an automated volumetric assessment of 45 cerebral structures while focusing on basal ganglia and thalamus. Successful automated segmentation was obtained in $98 \%$ of the participants, thus confirming the feasibility as well as the potential of fully automated morphometric approach with tools like MorphoBox in daily clinical practice. In addition, several studies have evaluated the morphological changes of basal ganglia after stroke [3, 8, 9, 19-25]. These studies alternatively used qualitative analyses [21, 22], morphometric methods with manual $[19,23]$ or semi-automatic delineation $[8,9,20,25]$, overall with a significant risk of segmentation error. None of these studies reported morphometric deviations (i.e., $Z$ scores) at the individual level, but only at the group level [8]. We thus report here, to our best knowledge, the first study evaluating morphometric changes at the patient level. This suggests good potential for automated brain morphometry to evaluate individual brain changes in patients with cerebrovascular diseases. 
Fig. 4 Correlations between stroke volume in the MCA territory and $Z$ scores of ipsilateral caudate nucleus, putamen, pallidum, and thalamus volumes
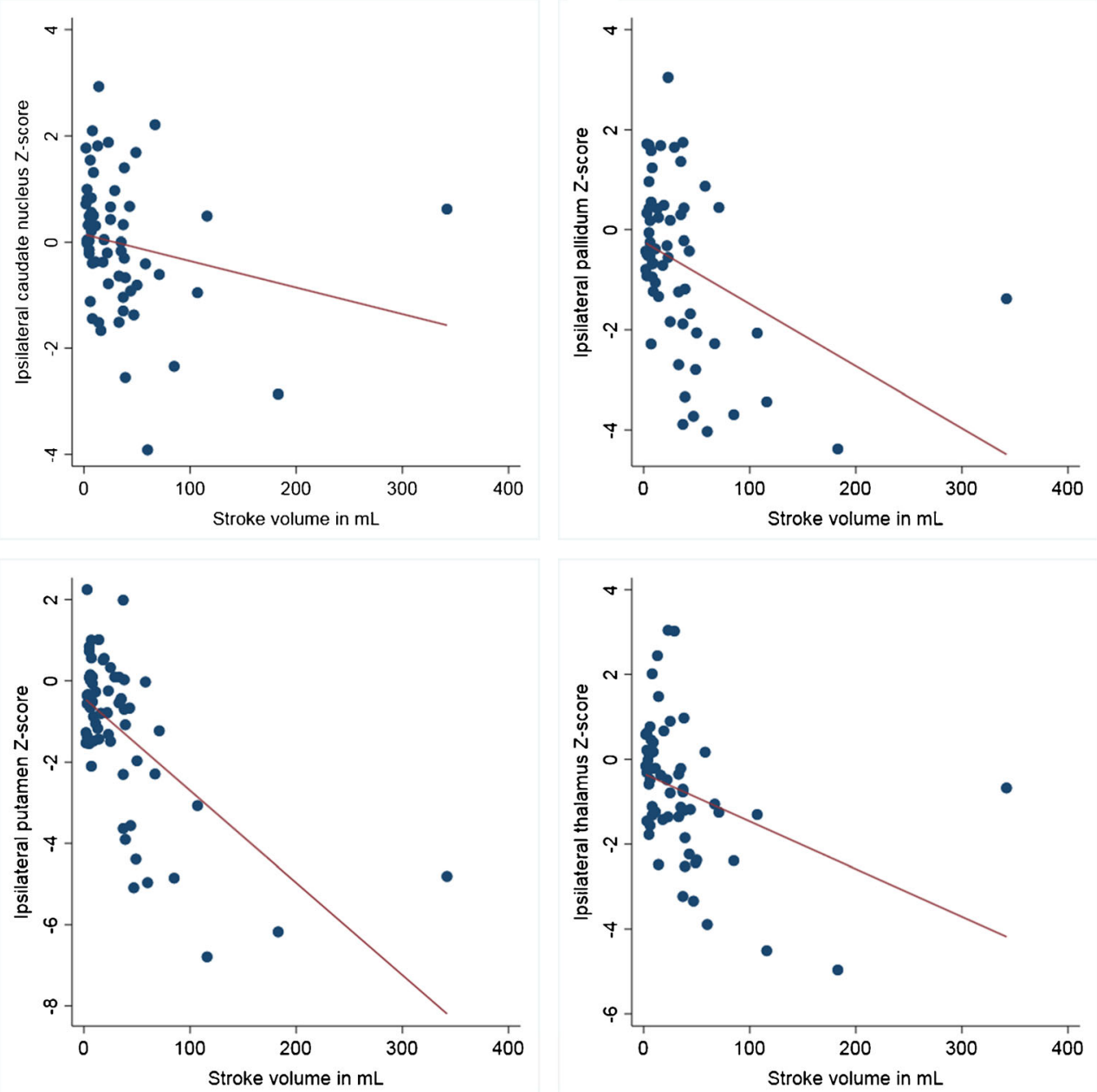

While we demonstrated that individual remote morphometric changes in chronic stroke can be assessed by automated segmentation, it remains unknown whether it could be done at the acute or subacute phase after stroke. Its potential to assess the time course of brain changes should hence be evaluated in further longitudinal studies.

\section{Morphometric changes in basal ganglia and thalamus after cortical stroke}

A significant change in basal ganglia volumes from brain reorganization following cortical stroke was expected [26, 27 considering the connection between the cortex and basal ganglia, and confirmed in the present study. Localization of initial cortical stroke significantly influences remote changes of basal ganglia and thalamus. We thus observed atrophy of the caudate nucleus, putamen, pallidum, and thalamus in patients affected by MCA stroke only. This is consistent with several studies that reported morphological changes [21, 22], putamen atrophy [20], or ipsilateral thalamus atrophy [9, 20, $23,25]$, indicating thalamic degeneration following proximal occlusion of the MCA. According to Tamura et al. [23], thalamic atrophy at the chronic phase was most likely due to a phenomenon of long-lasting retrograde neuronal degeneration rather than concomitant ischemia during stroke. Indeed, the thalamus and the MCA do not share the same vascular network, as the thalamus is mostly vascularized by the PCA. Regarding the caudate nucleus, results are more controversial, authors alternatively reporting a volume increase of ipsilateral caudate head [8], an absence of significant change in caudate nucleus volume [20], or an atrophy of the ipsilateral caudate nucleus [25]. Nevertheless, comparison with these studies is difficult. In order to specifically evaluate the morphometry of the striatum and thalamus, only patients with purely cortical stroke were included. On the contrary, in most studies published, patients with proximal MCA occlusion or combining all vascular territories were also included; therefore, some of these patients had ischemic lesions of the basal ganglia themselves, which precluded reliable analysis of the volumes of pallidum and putamen, and biased the thalamus and caudate nucleus analyses [19, 21-23, 25]. The first individual automated volumetric analysis of basal ganglia and thalamus at the 
chronic phase of a purely cortical stroke is reported by our study. While those changes depend on the vascular territory affected by the stroke, it remains unknown whether changes are influenced by some individual factors.

\section{Impact of stroke volume and cardiovascular risk factors}

Finally, beyond stroke location, it was possible to demonstrate that stroke extent had a significant linear impact on the trophicity of the central gray matter ipsilaterally to the affected hemisphere. The volume of cortical stroke negatively correlated with the extent of the morphometric changes of the caudate nucleus, putamen, pallidum, and thalamus in case of an MCA stroke. Tamura et al. [23] have also described that thalamic atrophy appears to be related to the size and location of the infarct, as patients with thalamic atrophy had a large fronto-parietal infarct in their study. In addition, although they did not evaluate the correlation between stroke volume and basal ganglia volume, Yang et al. [25] demonstrated a positive correlation between stroke volume and impaired motor function as well as a negative correlation between ipsilateral thalamus volume and impaired motor function, which suggested a negative relation between infarct and ipsilateral thalamus volumes. While we observed these changes at the chronic phase of a cortical stroke, there is little data on the exact kinetics of occurrence of these changes. Brodtmann et al. [9] suggested that thalamic atrophy could be observed as early as $3 \mathrm{~h}$ after the onset of symptoms. In a population of 125 patients who had surgery for resection of a cortical lesion, Kamiya et al. [28] observed transient diffusion restriction of the ipsilateral striatum and thalamus as early as 7 days after the intervention followed by atrophy of these structures; the extension of the anomalies being correlated with the extension of the resection. This indicates the presence of early morphological alterations of the interconnected structures within the striato-thalamocortical network ipsilateral to MCA stroke. Finally, we did not find any significant relation between cardiovascular risk factors, Fazekas score, presence of carotid artery stenosis $\geq$ $50 \%$, or delay from stroke onset, and striato-thalamic $Z$ scores after an MCA cortical stroke. Although this suggests that remote changes are independent of cardiovascular risk factors, some other factors such as endothelial function, genetic susceptibility to neuronal degeneration and neovascularization, or other metabolic function such as lactate production [29, 30] may have influenced the individual interrelation between stroke volume and striato-thalamic trophicity. In a transient 1$\mathrm{h}$ murine occlusion model of MCA, Hara et al. [31] demonstrated that atrophy of the thalamus was observed 1 month after the event and could be significantly reduced after administration of a calcium channel blocker, and a glutamate receptor antagonist, which indicated the presence of a transsynaptic mechanism in the neuronal degeneration of distant structures connected to the ischemic cortical zone (i.e., as the basal ganglia). In humans, the dynamics of basal ganglia alterations after cortical stroke should therefore be the subject of prospective longitudinal studies to determine the optimal time to initiate treatment with a view to stopping, or limiting the mechanisms inducing a cortical stroke neuronal degeneration, such as excitotoxicity, oxidative damage, apoptosis, inflammatory gliosis [27], or to promote mechanisms counteracting this degeneration such as neoangiogenesis [30]. The role of automated brain morphometry to help monitoring potential therapeutic effect could also be assessed.

\section{Study limitations}

Several limitations must be conceded in this study, the first being the small number of patients included with a stroke of the ACA or PCA territories. We did not find significant volume modifications or correlation between stroke volume and basal ganglia $Z$ scores, which could be due to lack of statistical power. Results for each of these vascular territories should thus be confirmed in a larger study population. Secondly, our retrospective study lacks longitudinal follow-up data from the acute to the chronic phase. This would be needed to access the exact kinetics of atrophy phenomena as well as to evaluate inter-individual atrophy rate variations. Both might be crucial to determine the optimal time point to treat remote neurodegeneration. Also, prospective longitudinal analysis including structural and functional connectivity mapping could provide a better understanding of potential compensatory networks after initial stroke. Finally, if morphometric changes have been observed, the relation between volumetric changes and patients' functional recovery should be evaluated; this was out of the scope of the current work.

\section{Conclusion}

Automated MR morphometry demonstrated atrophy of basal ganglia and thalamus at the chronic phase after cortical stroke in the MCA territory, this atrophy being related to stroke volume. These results confirm remote changes of basal ganglia and thalamus after cortical stroke and the potential role for automated MRI volumetry to assess brain plasticity after stroke. Whether automated MRI volumetry could be used to assess acute-subacute remote changes or brain salvage therapies efficacy at the patient level remains unknown and should be evaluated by further longitudinal studies.

Authors' contributions Cindy Baudat participated in data acquisition, statistical analysis, draft, and critical revision of the manuscript. Bénédicte Maréchal, Ricardo Corredor-Jerez, Tobias Kober, Reto Meuli, Patric Hagmann, and Patrik Michel participated in data acquisition and interpretation, and critical revision of the manuscript. Philippe Maeder participated in study conception and design, data acquisition, 
draft, and revision of the manuscript. Vincent Dunet participated in study conception and design, data acquisition, statistical analysis, draft, and revision of the manuscript.

Data availability The datasets used during the current study are available from the corresponding author on reasonable request.

Funding information The authors did not receive any financial support to conduct this study.

\section{Compliance with ethical standards}

Conflicts of interest Bénédicte Maréchal, Ricardo Corredor-Jerez and Tobias Kober are fulltime employees of Siemens Healthcare AG Switzerland.

Patrik Michel has received research grants from the Swiss National Science Foundation, the Swiss Heart Foundation and the ERISTA program (BMS/Pfizer), and consulting fees from Medtronic. All this support goes to his institution and is used for stroke education and research.

Cindy Baudat, Reto Meuli, Patric Hagmann, Philippe Maeder and Vincent Dunet declare that they have no conflict of interest.

Ethical approval All procedures performed in studies involving human participants were in accordance with the ethical standards of the institutional and/or national research committee and with the 1964 Helsinki declaration and its later amendments or comparable ethical standards.

Informed consent All collected data were anonymized to comply with national ethical guidelines and laws. Therefore, patients' consent was waived.

Open Access This article is licensed under a Creative Commons Attribution 4.0 International License, which permits use, sharing, adaptation, distribution and reproduction in any medium or format, as long as you give appropriate credit to the original author(s) and the source, provide a link to the Creative Commons licence, and indicate if changes were made. The images or other third party material in this article are included in the article's Creative Commons licence, unless indicated otherwise in a credit line to the material. If material is not included in the article's Creative Commons licence and your intended use is not permitted by statutory regulation or exceeds the permitted use, you will need to obtain permission directly from the copyright holder. To view a copy of this licence, visit http://creativecommons.org/licenses/by/4.0/.

\section{References}

1. Collaborators GBDN (2019) Global, regional, and national burden of neurological disorders, 1990-2016: a systematic analysis for the Global Burden of Disease Study 2016. Lancet Neurol 18(5):459480. https://doi.org/10.1016/S1474-4422(18)30499-X

2. Collaborators GBDS (2019) Global, regional, and national burden of stroke, 1990-2016: a systematic analysis for the Global Burden of Disease Study 2016. Lancet Neurol 18(5):439-458. https://doi. org/10.1016/S1474-4422(19)30034-1

3. Abela E, Missimer JH, Federspiel A, Seiler A, Hess CW, Sturzenegger M, Wiest R, Weder BJ (2015) A thalamic-frontoparietal structural covariance network emerging in the course of recovery from hand paresis after ischemic stroke. Front Neurol 6: 211. https://doi.org/10.3389/fneur.2015.00211

4. Kuchcinski G, Munsch F, Lopes R, Bigourdan A, Su J, Sagnier S, Renou P, Pruvo JP, Rutt BK, Dousset V, Sibon I, Tourdias T (2017) Thalamic alterations remote to infarct appear as focal iron accumulation and impact clinical outcome. Brain 140(7):19321946. https://doi.org/10.1093/brain/awx114

5. Visser MM, Marechal B, Goodin P, Lillicrap TP, Garcia-Esperon C, Spratt NJ, Parsons MW, Levi CR, Bivard A (2019) Predicting modafinil-treatment response in poststroke fatigue using brain morphometry and functional connectivity. Stroke 50(3):602-609. https://doi.org/10.1161/STROKEAHA.118.023813

6. Duering M, Righart R, Wollenweber FA, Zietemann V, Gesierich B, Dichgans M (2015) Acute infarcts cause focal thinning in remote cortex via degeneration of connecting fiber tracts. Neurology 84(16):16851692. https://doi.org/10.1212/WNL.0000000000001502

7. Cheng B, Schulz R, Bonstrup M, Hummel FC, Sedlacik J, Fiehler J, Gerloff C, Thomalla G (2015) Structural plasticity of remote cortical brain regions is determined by connectivity to the primary lesion in subcortical stroke. J Cereb Blood Flow Metab 35(9):1507-1514. https://doi.org/10.1038/jcbfm.2015.74

8. Abela E, Seiler A, Missimer JH, Federspiel A, Hess CW, Sturzenegger M, Weder BJ, Wiest R (2015) Grey matter volumetric changes related to recovery from hand paresis after cortical sensorimotor stroke. Brain Struct Funct 220(5):2533-2550. https://doi.org/10.1007/s00429-014-0804-y

9. Brodtmann A, Pardoe H, Li Q, Lichter R, Ostergaard L, Cumming T (2012) Changes in regional brain volume three months after stroke. J Neurol Sci 322(1-2):122-128. https://doi.org/10.1016/j.jns.2012.07. 019

10. Schmitter D, Roche A, Marechal B, Ribes D, Abdulkadir A, BachCuadra M, Daducci A, Granziera C, Kloppel S, Maeder P, Meuli R, Krueger G, Alzheimer's Disease Neuroimaging I (2015) An evaluation of volume-based morphometry for prediction of mild cognitive impairment and Alzheimer's disease. Neuroimage Clin 7:7-17. https://doi.org/10.1016/j.nicl.2014.11.001

11. Dunet V, Deverdun J, Charroud C, Le Bars E, Molino F, Menjot de Champfleur S, Maury F, Charif M, Ayrignac X, Labauge P, Castelnovo G, Pinna F, Bonafe A, Geny C, Menjot de Champfleur N (2017) MRI volumetric morphometry in vascular parkinsonism. J Neurol 264(7):1511-1519. https://doi.org/10. 1007/s00415-017-8561-5

12. Allen LM, Hasso AN, Handwerker J, Farid H (2012) Sequencespecific MR imaging findings that are useful in dating ischemic stroke. Radiographics 32(5):1285-1297; discussion 1297-1289. https://doi.org/10.1148/rg.325115760

13. Hammers A, Heckemann R, Koepp MJ, Duncan JS, Hajnal JV, Rueckert D, Aljabar P (2007) Automatic detection and quantification of hippocampal atrophy on MRI in temporal lobe epilepsy: a proof-of-principle study. Neuroimage 36(1):38-47. https://doi.org/ 10.1016/j.neuroimage.2007.02.031

14. Heckemann RA, Keihaninejad S, Aljabar P, Gray KR, Nielsen C, Rueckert D, Hajnal JV, Hammers A, Alzheimer's Disease Neuroimaging I (2011) Automatic morphometry in Alzheimer's disease and mild cognitive impairment. Neuroimage 56(4):2024 2037. https://doi.org/10.1016/j.neuroimage.2011.03.014

15. Jack CR Jr, Bernstein MA, Fox NC, Thompson P, Alexander G, Harvey D, Borowski B, Britson PJ, Whitwell JL, Ward C, Dale AM, Felmlee JP, Gunter JL, Hill DL, Killiany R, Schuff N, FoxBosetti S, Lin C, Studholme C, CS DC, Krueger G, Ward HA, Metzger GJ, Scott KT, Mallozzi R, Blezek D, Levy J, Debbins JP, Fleisher AS, Albert M, Green R, Bartzokis G, Glover G, Mugler J, Weiner MW (2008) The Alzheimer's disease neuroimaging initiative (ADNI): MRI methods. J Magn Reson Imaging 27(4):685-691. https://doi.org/10.1002/jmri.21049

16. Petersen RC, Aisen PS, Beckett LA, Donohue MC, Gamst AC, Harvey DJ, Jack CR Jr, Jagust WJ, Shaw LM, Toga AW, Trojanowski JQ, Weiner MW (2010) Alzheimer's disease neuroimaging initiative (ADNI): clinical characterization. Neurology 74(3):201-209. https://doi.org/10.1212/WNL.0b013e3181cb3e25 
17. Fazekas F, Kleinert R, Offenbacher H, Schmidt R, Kleinert G, Payer F, Radner H, Lechner H (1993) Pathologic correlates of incidental MRI white matter signal hyperintensities. Neurology 43(9):1683-1689. https://doi.org/10.1212/wnl.43.9.1683

18. May A, Gaser C (2006) Magnetic resonance-based morphometry: a window into structural plasticity of the brain. Curr Opin Neurol 19(4):407-411. https://doi.org/10.1097/01.wco.0000236622. 91495.21

19. Looi JC, Tatham V, Kumar R, Maller JJ, Millard E, Wen W, Chen X, Brodaty H, Sachdev P (2009) Caudate nucleus volumes in stroke and vascular dementia. Psychiatry Res 174(1):67-75. https://doi. org/10.1016/j.pscychresns.2009.04.002

20. Lopes MA, Firbank MJ, Widdrington M, Blamire AM, Kalaria RN, O'Brien JT (2012) Post-stroke dementia: the contribution of thalamus and basal ganglia changes. Int Psychogeriatr 24(4):568-576. https://doi.org/10.1017/S1041610211002195

21. Nakane M, Tamura A, Sasaki Y, Teraoka A (2002) MRI of secondary changes in the thalamus following a cerebral infarct. Neuroradiology 44(11):915-920. https://doi.org/10.1007/s00234002-0846-3

22. Ogawa T, Yoshida Y, Okudera T, Noguchi K, Kado H, Uemura K (1997) Secondary thalamic degeneration after cerebral infarction in the middle cerebral artery distribution: evaluation with MR imaging. Radiology 204(1):255-262. https://doi.org/10.1148/radiology. 204.1.9205256

23. Tamura A, Tahira Y, Nagashima H, Kirino T, Gotoh O, Hojo S, Sano K (1991) Thalamic atrophy following cerebral infarction in the territory of the middle cerebral artery. Stroke 22(5):615-618. https://doi.org/10.1161/01.str.22.5.615

24. Yassi N, Malpas CB, Campbell BC, Moffat B, Steward C, Parsons MW, Desmond PM, Donnan GA, Davis SM, Bivard A (2015) Contralesional thalamic surface atrophy and functional disconnection 3 months after ischemic stroke. Cerebrovasc Dis 39(3-4):232241. https://doi.org/10.1159/000381105
25. Yang M, Yang YR, Li HJ, Lu XS, Shi YM, Liu B, Chen HJ, Teng GJ, Chen R, Herskovits EH (2015) Combining diffusion tensor imaging and gray matter volumetry to investigate motor functioning in chronic stroke. PLoS One 10(5):e0125038. https://doi.org/10. 1371/journal.pone. 0125038

26. Haber SN, Calzavara R (2009) The cortico-basal ganglia integrative network: the role of the thalamus. Brain Res Bull 78(2-3):69-74. https://doi.org/10.1016/j.brainresbull.2008.09.013

27. Zhang J, Zhang Y, Xing S, Liang Z, Zeng J (2012) Secondary neurodegeneration in remote regions after focal cerebral infarction: a new target for stroke management? Stroke 43(6):1700-1705. https://doi.org/10.1161/STROKEAHA.111.632448

28. Kamiya K, Sato N, Nakata Y, Ito K, Kimura Y, Ota M, Takahashi A, Mori H, Kunimatsu A, Ohtomo K (2013) Postoperative transient reduced diffusion in the ipsilateral striatum and thalamus. AJNR Am J Neuroradiol 34(3):524-532. https://doi.org/10.3174/ajnr.A3242

29. Berthet C, Castillo X, Magistretti PJ, Hirt L (2012) New evidence of neuroprotection by lactate after transient focal cerebral ischaemia: extended benefit after intracerebroventricular injection and efficacy of intravenous administration. Cerebrovasc Dis 34(5-6):329-335. https://doi.org/10.1159/000343657

30. Yanev P, Seevinck PR, Rudrapatna US, Bouts MJ, van der Toorn A, Gertz K, Kronenberg G, Endres M, van Tilborg GA, Dijkhuizen RM (2017) Magnetic resonance imaging of local and remote vascular remodelling after experimental stroke. J Cereb Blood Flow Metab 37(8):2768-2779. https://doi.org/10.1177/ $0271678 X 16674737$

31. Hara H, Harada K, Sukamoto T (1993) Chronological atrophy after transient middle cerebral artery occlusion in rats. Brain Res 618(2): 251-260. https://doi.org/10.1016/0006-8993(93)91273-u

Publisher's note Springer Nature remains neutral with regard to jurisdictional claims in published maps and institutional affiliations. 